Voix et Images

\title{
Le Signe " silence " dans la poésie moderne. Application à quelques poèmes québécois
}

\section{Jean-Paul Mauranges}

Volume 3, numéro 1, septembre 1977

Nicole Brossard

URI : https://id.erudit.org/iderudit/200090ar

DOI : https://doi.org/10.7202/200090ar

Aller au sommaire du numéro

Éditeur(s)

Les Presses de l'Université du Québec

ISSN

0318-9201 (imprimé)

1705-933X (numérique)

Découvrir la revue

\section{Citer cet article}

Mauranges, J.-P. (1977). Le Signe « silence » dans la poésie moderne. Application à quelques poèmes québécois. Voix et Images, 3(1), 81-95. https://doi.org/10.7202/200090ar d'utilisation que vous pouvez consulter en ligne. 


\section{Le Signe «silence» dans la poésie moderne Application à quelques poèmes québécois ${ }^{1}$}

Existe-t-il de par le monde, quelque chose de plus fascinant que le silence? Que l'on évoque l'ordre humain - l'incommunicabilité de deux êtres qui n'ont rien à se dire, ou au contraire la communion des amants dans l'indicible - ou que l'on se tourne vers l'ordre cosmique - le silence de l'espace pour Pascal ou les scientifiques, où parle le Dieu de Moïse, celui aussi sans écho, que tente dramatiquement de sonder Job - ce vide total ou cette plénitude totale ont au moins un point commun: la tension extrême d'un espace qui, de toute sa force, tend à unir ou à éloigner, vers ce que les mathématiciens désigneraient cómme infini positif ou infini négatif.

La Poésie n'a pas, de tous les Arts, le privilège exclusif de susciter l'espace. La peinture avec la perspective, fût-ce en trompe-l'œil, l'équilibre ou déséquilibre des masses, la densité ou transparence de la couleur, etc., s'étend déjà sur deux dimensions et cherche souvent à rendre l'illusion d'une troisième. L'architecture est éminemment créatrice d'espace: songeons à une flèche de cathédrale, un stade olympique, un jardin bouddhique Zen. Mais la poésie, plus que tout autre art peut-être, hormis la musique et la danse, possède la faculté de créer des intervalles. Parce que déjà, par nature et par vocation, elle est relation posée entre deux énoncés, et qu'elle a ce pouvoir étonnant de faire surgir un sens qui n'est pas, comme en prose, la somme des deux premiers. La peinture peut se suffire d'un trait, d'une tache de couleur. De même, l'architecture peut-elle renoncer à la création d'intervalles: un obélisque, un barrage, une pièce d'eau ont en eux-mêmes leurs lignes de fuite. En revanche, on imagine mal qu'en Poésie un mot isolé, fût-il "soleil» ou «nuit» ou "violon" puisse en soi, être poétique. Il lui faut un correspondant, de consonance ou de dissonance, pour, non seulement "dire" quelque chose (ce serait encore de la prose), mais surtout provoquer un écho. Ainsi a-t-on chez Nerval un «soleil noir», chez Baudelaire la "douce nuit qui marche», chez Verlaine les «sanglots longs/ des violons".

Le mystère poétique ne résiderait-il pas, pour une large part, dans cet écart entre les mots, et qui représente, dans l'espace et dans le temps, une fraction plus ou moins longue de silence? 
Par écart, on peut comprendre essentiellement une incohérence sémantique, et que celle-ci s'inscrive dans la «grammaire" de l'énoncé, (lexique et syntaxe), dans sa phonétique (prosodie) ou encore dans sa spatialisation (composition strophique, calligrammes et poésie concrète).

Jean Cohen (cf. Structure du langage poétique, Paris, Flammarion, 1966) relève cet écart de sens par exemple dans ce vers, apparemment si simple, d'Henri de Régnier: "Les feuilles chuchotent si bas..." et distingue deux étapes de l'effet poétique: une étape négative, la saisie du signifié (Sé, ), normalement dénoté par le signifiant (Sa), et qui, en l'occurrence ne convient pas (les feuilles ne «chuchotent» pas!); une étape positive, la réduction de l'écart, par la découverte d'un signifié (Sé2), qui est pertinent subjectivement, car il représente une connotation heureuse (le froissement de feuilles évoque un chuchotement). Jean Cohen donne le nom de métaphore à toute réduction d'écart. Henri Bonnard, qui commente cette analyse (in Étude du langage poétique, application à Guillaume Apollinaire, Paris, Éditions Europe, 1967, p. VI, 5) précise: "Ainsi le verbe chuchoter anime les feuilles, en fait des êtres échangeurs de secrets ou de commentaires sur la vie des hommes... Le Sé 2 n'est autre que le signifié attendu (celui de "bruissent "), enrichi d'une connotation qui affecte l'entourage. " C'est bien ce genre d' «impertinence» de sens, comme dit Jean Cohen, qui rend compte de l'étrange beauté de vers comme celui-ci, de Paul Éluard: «La terre est bleue comme une orange."

Ce type d'analyse est applicable au niveau phonique et prosodique. L'، impertinence " sémantique est présente alors même que l'on serait tenté de parler de parfaite cohésion. Soit ce vers célèbre de Racine: “Et les monts et la mer montent jusques au ciel» ou celui de Hugo: "Quand la lune apparaît dans la brume des plaines". Dans le premier cas, on a coutume de désigner cet effet de style sous le nom d'onomatopée. Dans le deuxième cas, ainsi que le fait remarquer $H$. Meschonnic (cité par A. J. Greimas, Essais de sémiotique poétique, Paris, Larousse, 1972, p. 29) «on peut enregistrer une symétrie phonique presque parfaite et dire alors que "le retour de la même série de voyelles dans les deux hémistiches... tient le vers!" Mais à y regarder de près, dans le premier exemple, il y a moins imitation que transposition de son. L'harmonie n'est pas ici imitative, mais analogique. En fait, elle est arbitraire, mais aussi évocatrice de connotations plausibles. Le son « $\mathrm{m} »$ n'est en rien contraignant, ni même en soi suggestif: que l'on songe, pour s'en convaincre, à ce tableau moderne de Duchamp intitulé : «Tu $m$ '!". Le symbolisme ressortit donc d'une réduction de l'écart entre un premier signifié, évident mais banal (on dirait que les monts montent vers le ciel) et un second signifié qui, lui, est riche de sens (amplification sonore, visuelle, dramatique, symbolique, etc.). Dans le deuxième exemple, l'homogénéité phonique est une consonance prosodique certes, mais une telle équivalence au niveau acoustique n'agit pas comme répétition de son et de sens, mais comme infime variation sonore (en raison du changement de consonnes) et par conséquent comme infime mutation de sens; tandis qu'en prose, ce vers se lirait avec onze pieds 
(brum'), ici il doit être lu en tant qu'alexandrin. L'articulation phonique qui, en prose, serait passée inaperçue et aurait donné un énoncé banal, reçoit, de par sa cadence, son équilibre d'harmonie, une signification nouvelle: la lune ne fait pas qu'apparaître quelque part, elle apparaît a:ec une douceur diffuse. En bref, ce qui pouvait être dénoté comme harmonie et musique doit être maintenant connoté comme charge harmonique et musicalité.

La notion d'écart est encore plus manifeste dans ce qu'on pourrait appeler la géométrie du poème, qu'il s'agisse de composition strophique de type classique ou d'expériences de visualisation concrète comme l'ont tentée Apollinaire avec ses Calligrammes ou les lettristes modernes (suisses comme Gomringer, allemands comme Mon, français comme Bory, etc.). A ce titre, il peut être utile de rappeler ces remarques du philosophe autrichien Ludwig Wittgenstein: "La langue ne peut pas représenter ce qui se réflète en elle. Nous ne pouvons pas exprimer par la langue ce qui s'exprime dans la langue. »C'est dire à la fois les limites de la langue mais aussi l'infinie possibilité de création qui lui est inhérente, dès lors que les relations de cette langue avec l'univers ne sauraient être autres qu'à priori, arbitraires et toutes d'imagination. Mais les dadaïstes et les lettristes ont montré que cette imagination créatrice de mondes et, par là même, génératrice de communication entre auteur et lecteur, n'avait pas à se fonder exclusivement sur une langue associative, où le mot est évocateur d'images, soit par comparaison, soit par assimilation: «Comme un long linceul traînant à l'Orient" (Baudelaire), “La rosée aux doigts de chat" (A. Breton) ou par un système phonologique de mise en relation: "Aboli bibelot d'inanité sonore» (Mallarmé). Le spatialisme introduit un type original de communication: «la langue se présente devant le poète comme une «matière" à exploiter poétiquement, matière plus ou moins dense, ensemble de signes plus ou moins espacés, plus ou moins énergétiques. Tout devient alors possible: la réduction des mots, leur atomisation, le travail des souffles et des articulations, la danse des lettres, la création de signes nouveaux, le montage de mécanismes linguistiques" (Pierre Garnier, Spatialisme et poésie concrète, Paris, NRF, 1968, p. 12). L'information ne vise plus l'intelligibilité du "texte", ni même la seule innovation esthétique du "métatexte» (on aurait de la peinture abstraite et non de la poésie) mais les relations entre un "texte" désormais non contraignant quant à son sens et un langage insolite, fait de signes qui ne sont plus «attactiés à un quelconque signifié mais sont signifiants en eux-mêmes" (p. 20). Ou, pour résumer la chose, avec Max Bense, on peut dire qu'il y a «isomorphie linguistique" (mais aussi poétique) - «entre le texte et le métatexte» (p. 15). Puisque, même dans ce genre où l'énoncé semble se volatiliser, il y a évidemment dialogue entre les signes, on se doute que l'élément «espace " le plus chargé d'intensité de «communication» (essentiellement énergétique, cybernétique) sera le «blanc» typographique; il correspond à ce qu'en langage pictural on a appelé des «tensions d'espace" (P. Dufayet, $Y$. Jenger, le Comment de la poésie, Paris, Éditions ESF, 1973, p. 84). 
On voit que dans tous les cas la notion d'écart est capitale. Pourtant, il semble que l'on n'ait jusqu'à présent pas accordé assez d'importance à ce signe d'écart par excellence qu'est le silence.

\section{LE SILENCE}

Venant comme intervalle dans une suite linéaire de mots chargés, les uns pour les autres, de sens, il est d'abord un signe supplémentaire de sens, soit comme prolongement ou anticipation, soit comme rupture du message, discontinuité du sens. Puisque le poème est également un acte inscrit dans une série temporelle, le silence intervient comme liaison ou pause, halte ou saccade, hésitation ou fuite, bref comme élément structurel du mouvement du poème. Enfin il apparaît sur le papier comme un blanc sur blanc et noir, et l'on comprend qu'il puisse agir pour renforcer l'une ou l'autre de ces dominantes. C'est pourquoi on peut étudier la force du silence sous ses trois aspects: sa signification dans l'ordre du dire poétique, sa dynamique et enfin sa géométrie.

II est évident que l'éclatement du vers classique, l'avènement des formes libres ont favorisé l'épanouissement du silence, jusque-là comprimé dans quelques hardiesses de style qui ont pour nom: le hiatus euphonique (en général soigneusement évité en prosodie traditionnelle), la césure, l'enjambement, le rejet, ou encore limité à une graphie rudimentaire de ponctuation, dont les signes les plus courants sont le tiret, les points de suspension et d'exclamation. Aurait-on jamais osé, avant les surréalistes, employer la parenthèse, un mot réduit à une lettre et un apocope ? Eut-il été imaginable avant Apollinaire, ce délicat poème en gouttes d'encre d'imprimerie pour représenter la chute de pluie que filtre la lumière, de papier? Le lettrisme n'est-il pas une forme extrême du silence non figuratif, par excellence non signifiant, une sorte d'infini négatif de l'acte de parler et d'écrire?

Tentons de suivre jusqu'à ses avatars extrêmes, cette riche variation du silence en poésie moderne. Si l'on admet que le silence est un signe chargé de sens, on lui trouve essentiellement deux fonctions antithétiques: d'un côté il est une vibration prolongée des mots qu'il est chargé, par cette sorte de résonance, de voix intérieure, de mettre, sinon en accord ou harmonie, du moins en concordance: c'est l'accord supra-logique des mots qui ne sauraient être, sous peine de ridicule prosaique ou d'enfantement de monstres, reliés par une métaphore génitive ou une tournure hypotaxique ou au contraire coupés par une ponctuation intempestive:

Pleine joie sur les hommes

Et le cru de réveil qui monte de la rue

Voici naître la joie de haute certitude.

(Maurice Beaulieu, p. 112)

De l'autre côté, il apparaît comme déchirement, volonté d'anti-logisme comme par désespoir de trouver l'unisson. Ce silence est alors le rejet de notre conscience habituée, il est un choc, mais comme une sensation de 
vertige, d'attirance vers ce vide, ou plutôt ce trou béant dans nos habitudes de langage:

belles cadences girouettent dans la sacristie saccagée des images adieu, adieu je me tais désaffecté le carrousel halluciné du pur [poème

(Paul Chamberland, “le Temps de la haine», P. 261)

Dans le premier cas le silence apparaît comme une soumission attentive, à l'ordre nouveau de ces choses que l'on a dérangées de leur cohésion traditionnelle, (grammaticale ou lexicale), presque une marque de respect. Le poète ne se prétend ni démiurge ni apprenti sorcier. II se veut seulement le compositeur déférent d'une polyphonie nouvelle, certes, mais dont les éléments structuraux ne dépendent pas de lui; il assemble et met en harmonie ces éléments parce qu'au fond cette harmonie existe, elle est préétablie, semblait n'attendre que son interprète. Le silence est alors comparable à ce minime intervalle qui, dans la fresque vaticane de MichelAnge sépare Dieu de sa créature; le doigt de Dieu touchant presque le doigt de l'Homme: la séparation est en fait en même temps création de liberté, mais dans l'accord fondamental de son créateur. On trouverait chez PaulMarie Lapointe un bel exemple de cet affranchissement-jonction, qui n'a rien d'une liberté conditionnelle mais exprime la retenue de l'hypothèse chez le poète, en même temps que l'autonomie essentielle des objets que son langage unit:

j'écris arbre

arbre d'orbe en cône et de sève en lumière

racines de la pluie et du beau temps terre animée

(P.-M. Lapointe, "Arbres", p. 157)

Dans ce très long poème sur les arbres, P.-M. Lapointe développe un paysage habité par des objets (les arbres et ce qu'ils sont devenus, "coffres de fiançailles" ou "peuplier allumettes", ce qu'ils sont dans la forêt “captant l'oiseau captant le vent», et ce qu'ils représentent aux hommes, "les arbres sont couronnés d'enfants/tiennent chauds leurs nids/sont chargés de farine $")$. Or ce paysage, pour être étrangement composé, n'a rien d'arbitraire. Imaginons par contre des liaisons logiques, une ponctuation, des accords linguistiques: le lyrisme épique de cet hymne s'épuiserait dans un style de catalogue, d'arpenteur-géomètre comme aurait dit Giraudoux. La logique aurait mis en évidence un dictionnaire de l'absurde où des réalités se seraient trouvées en contact aberrant les unes avec les autres. Le silence qui, au contraire, aère de non-concordance cette immense suite parataxique fait ressortir la dépendance suprasensible et immanente de ces choses. Les objets ne sont plus mis bout à bout dans une chaîne fortuite ou expérimentale, mais sont entre eux en relation d'être.

Cependant ce type de silence, que l'on pourrait qualifier de choral, car il unit plusieurs voix ou plutôt échos, ne signifie pas nécessairement émerveillement et joie; il peut être tragique et les choses qu'il relie sont des lambeaux, tandis qu'apparaît dans toute sa trace déchirante la rupture des notations du réel: 
Le chant du seul est bref

Ët revient aussitôt

(Les ... ne sont pas de l'auteur)

Vers le seul en silence

et il meurt

Et le crépuscule se trouva

Libre

(Les ... sont de l'auteur)

Même le silex avait

Saigné

Tout I'horrible jour

(Wilfrid Lemoine, p. 136-137)

La mer n'en finit plus de mourir

Parle

Et tu mourras aussi

(Fernand Dumont, "le Gond de la mer ", p. 136)

Que ce soit par ces procédés des points de suspension, de la mise en retrait, de l'enjambement, de la suppression de la ponctuation, de la mise en abyme (jeu de miroirs ou de paraboles répercutant une image ou un écho), de l'ellipse, ces poèmes soulignent l'impérieuse nécessité du silence conducteur de compréhensibilité.

Peut-être doit-on ici remarquer que cette compréhensibilité a tendance à s'émanciper plus dans le sens de la dissonance que celui de la consonance? Ce qui ne veut nullement dire que les poèmes modernes soient plus amers et déchirants que les classiques, ou encore que l'impression de souffrance l'emporte sur celle de la joie. Par dissonance entendons seulement un écart de type linguistique. Par exemple, dans ce poème de Rina Lasnier, il y a silence entre le titre "Il suffit..." et la suite du récit:

D'un caillou pour moudre l'insecte

D'une main pour vaincre l'animal

D'un talon pour mépriser la poussière

D'un hallier pour étouffer le frère

D'un sein fermé pour haïr l'enfant,

Mais toi, Dieu, pour m'anéantir

Tu me broies sous la pierre de Christ.

(Rina Lasnier, “ll suffit...», p. 66)

Mais le silence est en concordance avec le message. Tout comme en polyphonie classique, le thème est annoncé, mis en résonance suspensive, repris par le développement (les cinq vers qui en sont le contrepoint), et après un autre silence-liaison (la virgule et l'intervalle), vient le renversement du thème dans la coda qui amplifie un des motifs du contrepoint (un caillou devient la pierre de Christ)

C'est également le cas dans ce poème d'André-Pierre Boucher:

Cette passerelle de brume

à moi seul possible

(... pour éviter les prochaines trahisons)

passerelle par-delà l'océan

(André-Pierre Boucher, «Donnez-moi », p. 244) 
Ici le décalage des vers et l'incise entre parenthèses sont comparables musicalement parlant, à l'insertion d'une altération, d'un chromatisme. Mais, fondamentalement, le mode et le système tonal - traduisons: la syntaxe et le lexique de base - sont respectés.

En revanche examinons ce poème de Claude Gauvreau:

Clic... Ic... Bric... Tipc...

Mon siège fend sur le travers,

L'aboiement né...

Oncle.

Pitrafe.

Je suis Néron...

Eil oreille ardoise

pas de pitié

pour Zo Mécu

L'offrande reste.

Parquez dans la Lavende.

Venez à moi

chères hirondelles.

Mon nom est Pétrafor.

(Claude Gauvreau, «Brochuges », p. 118-119)

De même que l'ordre linguistique est bouleversé, de même l'agencement phonique est-il aussi malmené par l'intention de violenter la langue dans tout son être. II en va en art comme en religion: l'iconoclaste vrai, n'est pas celui qui brise les icones, mais les idoles. II n'est pas un vandale pour le plaisir sadique de l'anarchie. II crée une nouvelle représentation sensible d'un monde, jusque-là insoupçonné, mais imminent. II le suscite avec un langage qui, malgré son caractère déroutant, reste identifiable: "Pitrafe" n'a certes pas droit de cité dans le Robert. Pourtant il n'a rien d'anglais ou de hongrois. II reste français, dans sa sonorité du moins. "Clic... Ic... Bric... Tipc..." également (un Allemand aurait choisi par exemple Hops... Zak, etc. !!) Pourtant, ce qui est radicalement changé, c'est l'axe directionnel de la compréhensibilité. Tandis que les poèmes cités plus haut, en dépit de la facture moderne, ont un sens (intelligibilité) parce que de l'alliance, même surprenante, de deux mots apparemment incompatibles naît un signifié plus pertinent que la somme des deux, ici l'auteur renonce délibérément à toute mise en relation de type associatif (signifié ${ }_{1}+$ signifié $_{2} \rightarrow$ signifiés$_{3}$ ). II opte pour une chaîne verbale désarticulée qui n'obéit plus aux lois combinatoires du langage, même le plus déconcertant. La révolution - et je ne dis pas qu'elle vienne plus de Gauvreau que d'un Apollinaire, d'un Henri Michaux ou d'un Gaston Miron - réside en ce que la nouvelle série (car l'expérience a quelque chose de comparable aux «séries" de la musique dodécaphonique) ne réagit plus à une intention fondamentale, une volonté de dire (sentiment ou une expérience, ou tout autre), elle n'est plus soumise à une pensée directrice, un rêve conducteur, une représentation du monde au besoin aberrante, mais logique dans son déroulement et finalement déchiffrable. Elle n'est plus liée à ce qu'on appelerait en musique une "tonique".

Le nouvel ordre créé procède d'un jeu d'à priorisme où l'expressivité provient, non plus d'une combinaison de mots et d'images, eux-mêmes 
générateurs de nouvelles images, mais d'une structure formelle posée à priori comme expérimentale, chargée d'explorer les ressources infinies de la communication. Celle-ci n'a plus à être plausible mais à évoquer des possibles. Si le philosophe autrichien L. Wittgenstein a dit: "les limites de ma langue sont les limites de mon monde", le poète est justement celui qui force ces frontières linguistiques. À la notion de compréhensibilité de type consonance, au besoin impertinente, rappelons-le, s'est substituée une compréhensibilité de type dissonance, mais telle que le nouveau langage doit déboucher finalement sur une nouvelle pertinence: faute de quoi le poème ne serait plus que puzzle facile et stérile. De même qu'en peinture il ne suffit pas de griffonner ou de barbouiller à l'aveuglette pour produire un Miro, un Dali ou un Vasarely, de même qu'en musique il ne suffit pas de combiner des enregistrements sur magnétophone, pour atteindre à l'expressivité d'un Messiaen, d'un Stockhausen ou d'un Boulez, ainsi en est-il de la poésie expérimentale, et en particulier lettriste; celle-ci ne s'amuse pas à secouer les pages d'un dictionnaire pour composer un rébus dont seuls des experts pourraient interpréter l'art vaticinatoire. Cette poésie a aussi ses lois (de substitution, de redondance, d'ellipse, etc.) et c'est là qu'intervient précisément une des plus précieuses: le silence. Certes il faut prendre ce mot dans un sens large. On peut concevoir plusieurs formes et fonctions de ce silence: il peut être une réduction minimale d'une phrase; ainsi le "tu m'.!» de Duchamp, qui, certes, n'a rien de poétique. Mais il en va certainement autrement de "Le meur/Le meurtre" dans le poème de Gauvreau: le silence morphologique est ici générateur d'une sémantique pathétique, mais non ostentatoire, non déclamatoire. Elle exprime, par la seule privation de quelques lettres, une suppression de vie. Inversement le silence peut être "maximaliste", dans la mesure où, grâce à lui, ce qui paraissait futile devient soudain essentiel. La remarque vaut bien pour les poèmes modernes de facture encore "figurative", soit que le silence soit initial:

II suffit...

dans le poème de Rina Lasnier,

médian :

L'éternité

se détache

de l'homme.

(F. Ouellette, «50 Mégatonnes», p. 189),

ou final :

La terre cesse d'être ronde

Pour leur indiquer

Où aller

Et elle s'étend comme

Une pelure d'orange

pelée (Jacques Godbout, "Comme une orange ", p. 236)

Cette révélation de l'essentiel par la plage de silence est encore plus nette quand, dans le poème "non figuratif», il n'y a apparemment rien à comprendre, rien à évoquer. Revoyons ce poème de Gauvreau: 
Les mains nues

ils ont marché

Les mains

Le meur

Le meurtre!

Marchés tout seuis

Le canon tonne........
(Les ... ne sont pas de l'auteur)

(Les ... sont de l'auteur)

Dans ce dernier vers le silence en pointillé est bien une salve, donc ressortit encore de l'art figuratif. Mais dans le précédent, ce silence semble injustifié, aussi vide de sens que la "faute" orthographique (marchés) qui le précède. Or c'est précisément là l'essentiel: ce vide de sens, qui soudain, devient étrangement expressif. La mort n'est-elle pas vide de sens, une faute d'orthographe, un trou absurde? L'aventure humaine qui reçoit cette marque du pluriel et du passif (marchés) n'est-elle pas une illusion collective et un destin subi? Le sens, ici, n'est pas aussi évident que dans ce vers de V. Hugo, avec la puissance du silence-rejet:

\section{Il s'appelait - Le Pauvre}

Mais on ne saurait mettre en doute sa faculté émotionnelle, peut-être d'autant plus vive qu'elle est inexplicable.

On peut donc provisoirement conclure que le silence, qu'il soit maximaliste ou minimaliste a une valeur vocative: c'est une fonction d'appel de l'expressivité.

Un facteur important d'expressivité est ce qu'on a, de tout temps, appelé la musicalité du poème. Parler de musicalité en poésie n'est pas qu'une métaphore désignant une sorte de musique purement intérieure. Si cette impression existe, c'est qu'elle est donnée par une phonologie repérable, une prosodie - même si cette prosodie est, en poésie moderne, très difficile à discerner, depuis qu'ont été oubliés la scansion, le mètre, la rime, etc. Or il est indéniable que la poésie moderne, qu'elle charme ou heurte notre oreille, est encore largement une musique, avec ses modulations, son rythme. Ce n'est pas à dire que l'analyse scientifique pourra nous livrer les secrets de la résonance affective ou symbolique qu'elle provoque. Du moins pourra-t-elle déjà repérer quantitativement les éléments probables d'une qualité sonore et, au-delà peut-être, nous guider dans l'appréhension des relations son et sens.

Des expériences très instructives ont été faites de stroboscopie phonétique de poèmes. Certes l'expérience garde sa part de subjectivité, puisqu'il faut d'abord que les poèmes soient récités et que la diction du récitant est, bien sûr, fonction de son “émotion ". En fait, on peut parfaitement concevoir qu'un ordinateur programmé sur la valeur acoustique moyenne des phonèmes d'une langue donnée transcrirait une image objective des agencements sonores d'un poème. Nous n'en sommes pas là. Et encore une fois, rien ne permettra de traduire une fréquence, une durée et une intensité, tous éléments physiques mesurables de la phonie, en qualité poétique. Du moins des corrélations entre phonétique et prosodie sont- 
elles riches d'enseignement. On a pu ainsi vérifier expérimentalement la musicalité des vers de Baudelaire - dits par Marguerite Moreno - où la durée de l'émission des syllabes est calculée en centièmes de secondes: (nous suivrons ici la description qu'en donne H. Bonnard, op. cit., p. II, 3 suiv.):

J'ai long temps / ha bi té // sous de vas / tes por ti-ques
$26 \quad 42 \quad 46$
212541
$14 \quad 3264$
$28 \quad 3858$

Tandis que la description traditionnelle - voyelles longues et courtes aurait donné une image statique d'un vers en quelque sorte isochrone, soit quatre anapestes (UU $\boldsymbol{*}$ ) et aurait de plus, placé la coupe du deuxième hémistiche après "vastes", la décomposition phonétique oblige à lire ce vers selon «un rythme dynamique, fait d'élans successifs exprimés par des éléments croissant en durée" et à couper au milieu du mot "vastes", donnant une «coupe enjambante" (H. Bonnard, p. II, 4). Certes, on ne peut tirer de conclusions générales de la valeur significative des phonèmes, surtout quant à leur valeur symbolique: songeons au symbolisme particulièrement heureux, mais en soi arbitraire, du traitement des voyelles par Rimbaud :

A noir, E blanc, I rouge, U vert, O bleu: voyelles...

Pourtant, il est exact que non seulement les voyelles peuvent se classer d'après leur sonorité en claires et graves, selon qu'elles sont antérieures ou plus ou moins fermées; elles correspondent assez souvent à des impressions sensibles, surtout une fois mises en relation avec un sens. C'est ainsi que l'on peut ressentir subjectivement les sons aigus de ce vers de Lamartine:

Le fifre aux cris aigus, le hautbois au son clair.

Une telle analyse phonématique, si elle n'a rien de rigoureux ni de contraignant pour l'interprétation sémantique, aide ainsi à mieux comprendre les articulations harmoniques d'un mot ou d'un vers. Et, pour le sujet qui nous occupe, on retiendra la distinction faite par P. Delattre ( «les Attributs physiques de la parole», in Revue d'esthétique, 1965, XVIII, p. 3-4) entre “inharmonie", ou bruit durant la tenue de la consonne (comme $K, t, p$ ) et "l'harmonie", ou absence de bruit (comme $r, j, l, w$ ) et la relation qui existe entre ces deux concepts: "La consonne $r$ qui, après une voyelle finale comme dans "fleurs» s'efface en de douces harmoniques", se renforce en un bruit sourd et rugueux lorsqu'elle suit une consonne dévoisée comme dans “cri » (P. Delattre, op. cit., p. 251).

Le silence, de la même manière, est repérable. Faute d'ordinateur, tentons, au moins empiriquement, de distinguer principalement deux types de silence: le silence harmonique, qui vient en prolongement d'un son, et le silence isolant, qui agit comme une césure, une rupture de son. L'un et l'autre pourront, selon le contexte sémantique, avoir un effet dissonant ou consonant. Examinons ce poème, «la Voix de l'oiseau », d'Anne Hébert:

J'entends le bruissement des peupliers

Qui font un chant liquide 
Tout autour de moi,

île noire

Sur soi enroulée.

Captivité.

(Anne Hébert, "la Voix de l'oiseau ", p. 79)

En dépit de la virgule qui semble unir les deux dernières strophes, le blanc qui les sépare, ainsi que le hiatus vocalique entre "moi" et "île", mots qui s'opposent en outre par leur valeur phonologique, tout concourt à créer un silence de type isolant. Dans le cas présent, il se justifie par l'idée que le passage se fait d'une impression auditive extérieure (le bruissement des poupliers) en une intériorisation de la sensation, mais en même temps, il s'avère que les cercles concentriques distincts ("Tout autour de moi" "Sur soi enroulée») ne sont pas étrangers l'un à l'autre; ils sont unis selon une relation paradigmatique, puisque le "soi" de la dernière strophe est bien sûr équivalent au "moi " de la précédente. A la phonie liquide du "autour de moi" correspond d'assez près "île noire/Sur soi enroulée». Un silence, toutefois, intervient entre "île noire" et "Sur soi enroulée", à valeur harmonique, comme on le voit, tandis qu'un autre silence, cette fois isolant, coupe les deux vers "Sur soi enroulée" et "Captivité".

II n'est pas besoin, pour susciter l'impression de silence, de s'en remettre à la seule verticalité du poème. L'écriture dans le sens horizontal permet un effet du même ordre. Des poètes comme Paul-Marie Lapointe ou Pierre Perrault (cf. Gélivures, Montréal, Hexagone, 1977) usent à bon escient du silence dans une suite linéaire horizontale. Prenons par exemple le poème déjà cité de P.-M. Lapointe, «Arbres»:

j'écris arbre

arbre pour l'arbre

bouleau merisier jaune et ondé bouleau flexible acajou sucré bouleau merisier odorant

rouge bouleau rameau de couleuvre feuille-

engrenage vidé bouleau cambrioleur à feuilles de

peuplier passe les bras dans les cages du temps captant l'oiseau captant le vent

bouleau à l'écorce fendant l'eau des fleuves

bouleau fontinal fontaine d'hiver jet figé bouleau des parquets cheminée du soir galbe des tours et des bals

albatros dormeur

(P.-M. Lapointe, «Arbres », p. 158)

Les silences "visuels" que nous avons déjà signalés ne sont pas là seulement pour éviter une ponctuation ou une articulation grammaticale qui seraient de toute façon superflue, sinon néfaste. Ils sont également autre chose qu'un simple procédé de lisibilité pour "aérer " le poème. Ils représentent manifestement une suite mélodique de silences harmoniques qui rendent perceptibles l'alternance régulièrè de voyelles semi-claires (é) et semi-graves (o), elles-mêmes en alternance avec des "demi-teintes» consonantiques, puisque, selon le classement de Delattre les consonnes $f, b$ se situent presque au milieu de l' «inharmonie" tandis que $m, r, j$, se trouvent presque au milieu de «l'harmonie». On dira peut-être que c'est un effet du 
hasard! Reconnaissons qu'il est heureux. C'est en tout cas un plaisir supplémentaire qu'a le lecteur attentif de pouvoir comprendre pourquoi il a trouvé, de prime abord, ce passage harmonieux. A la joie des yeux et de l'esprit face à des images qui s'ordonnent, comme ici, pour une vision et une sensation (gustative, olfactive, tactile) s'ajoute une joie pour l'oreille, et pour l'esprit encore, d'une phrase où sont mis en sympathie et en vibration des silences harmoniques.

La distinction entre "silence harmonique et isolant" n'est pas nécessairement fonction de l'écriture visuelle, des «blancs» du poème. Pour nous en convaincre, nous pouvons penser entre autres, aux poèmes de Raoul Duguay; ce dernier, lors d'une entrevue avec Richard Giguère, avouait: "Je suis un écrivain de l'oreille... C'est la vibration qui m'intéresse comme la source même du poème, c'est le pouvoir énergétique, le potentiel énergétique qui est contenu dans certains types de sons... Un éclatement vers la transparence: le réservoir du SILENCE" (in Voix et images, vol. I, $n^{\circ} 2$, décembre 1975, p. 157-170). Examinons ce poème de Duguay:

peut-être était-ce en hiver Les yeux de source déjà

muaient en

figée se

miroirs pour

confondre mon âme quelqu'un a échappé un

caillou et tout a

cassé tu étais pourtant incassable et

l'eau de source

fragile comme

(R. Duguay, Ruts, Montréal, L'Aurore, 1974)

Déjà le texte frappe par son consonantisme chargé d'occlusives sourdes [k] et cette sonorité a l'air d'être accentuée par la spatialisation: la densité des trois vers où dominent ces occlusives est comme mise visuellement en relief. Le poème est, de plus, traversé de silences, de type harmonique (précisés sémantiquement par le mot “se muaient») et de type isolant (cf. le mot "figée"). Mais ils ne suivent pas servilement la disposition typographique. II est clair que le silence qui distingue «déjà " et «figée» n'est pas du même ordre que celui qui semble relier "figé " à "muaient" ainsi qu'à «miroirs». En dépit de la rupture voulue entre «tout a»/et “cassé», il n'y a pas en fait, discontinuité, alors que celle-ci est manifeste entre "cassé " et «tu étais pourtant incassable". Enfin, s'il y a isolation entre deux groupes de mots (déjà/figée - incassable et/fragile), on constate que ces deux silences isolants sont en réalité en contrepoint, ne serait-ce que par leur consonantisme.

Si le silence graphique n'est donc pas nécessairement lié à une intention phonétique, il a néanmoins tendance à occuper une place de plus en plus grande en poésie moderne, sans parler de la poésie spatiale. Ce qui n'est, au fond, que justice, non seulement parce que le poète se doit d'explorer toutes les ressources du langage; mais aussi parce que le poème n'est pas toujours destiné à être récité ou chanté. Dès lors, la spatialisation intervient un peu comme un suppléant de la phonologie (accentuation, 
intonation, rythme) et de la mimique. Mais c'est en poésie concrète que l'art de composer avec des plages de silence - les blancs du papier prend toute son ampleur. Peut-être aussi parce que la poésie expérimentale moderne tend à se spécialiser, c'est-à-dire à sonder jusqu'à l'extrême un champ de recherches, la poésie spatiale a-t-elle dû se distinguer de la poésie phonétique (ct. les poèmes-partitions de B. Heidsieck, les Sonies de $P$. Garnier, les Lautgedichte de F. Mon, les audio-poèmes d'H. Chopin). Elle a voulu, elle aussi, à sa manière, organiser l'art des espaces, que ce soit dans la réalisation de microstructures à partir de lettres ou mots apparemment éparses et désordonnés, de type mécanique, ou dans l'ordonnance de poèmes visuels, où, comme le dit $P$. Garnier, "le visible englobe le lisible" (op. cit., p. 64). Dans tous les cas, le blanc intervient comme tension entre les lettres, les mots ou le graphisme, mais il n'est pas toujours possible de le désigner comme silence. Il est le plus souvent un «espaceénergie (ibid., p. 44) à valeur cinétique, comparable à ce qu'ont recherché, en musique, un Weber, un Stockhausen ou un Boulez, en art plastique, un Pollock ou un Vasarely. Évoquant le «poème sémantique», c'est-à-dire le poème «signal " et "signe d'une action», P. Garnier note justement: «La langue observe un absolu silence esthétique mais c'est elle qui désigne, qui dirige les gestes... C'est par la succession des blancs que le poète régularise, règle, automatise ou relâche le mouvement qu'il imprime " (op. cit., p. 109). Á la limite, il semble que même la notion de silence se volatilise, puisqu'il n'est plus de l'ordre de l'expression, encore moins de l'explication, mais fonctionne, comme le poème tout entier "à la manière d'une boussole qui indiquerait différentes directions» (op. cit., p. 112).

En dehors de ces frontières extrêmes, on peut encore identifier le silence «spatial " comme silence expressif, et non comme axe directionnel, voire multidirectionnel. En l'occurrence, il est vrai qu'il sera en deçà de la «matière sémantique» décrite par P. Garnier, puisqu'il ne portera pas en lui-même sa signification et sa force d'expressivité, mais l'indiquera en relation avec des lettres ou des mots. Nous en donnerons deux exemples: L'un du Père Sylvestre Houédard:

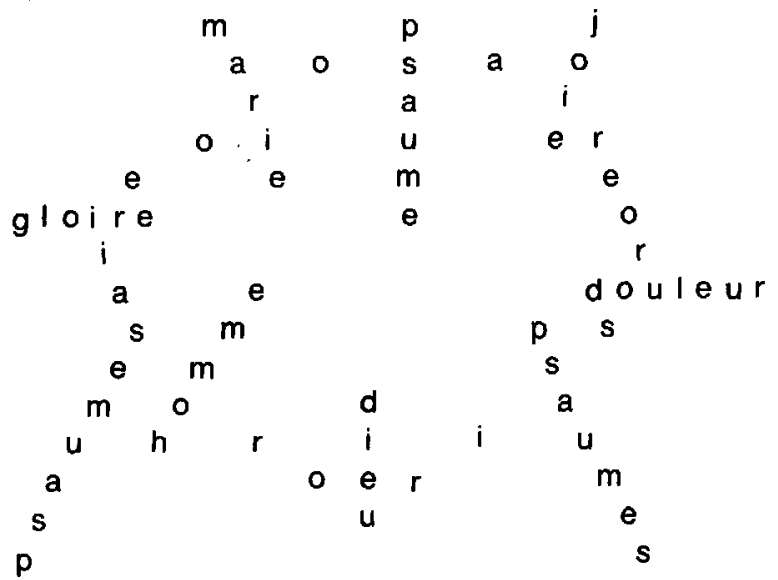


Nous $y$ lisons un message à la fois d'invariants (gloire - douleur), d'anamorphoses (psaumes) et de variables presque atomisées, bien qu'encore repérables (joie - douleur). Le silence n'est pas tant dans ce qui lie ou définit les lettres que dans l'absence de certaines lettres, à dessein: on constate ainsi que la lettre «l», cette liquide qui désigne autant «gloire" que «dou/eur" est absente de toutes les autres combinaisons spatiales. Et, chose curieuse, mais significative, lui est substituée une autre liquide, la lettre " $r$ " qui est certes présente dans les deux mots précités, mais aussi dans le mot de «Marie"; ce mot devient par là même le vocable générateur de tout le poème, alors qu'il ne lui était pas décerné, spatialement, une place prépondérante. Et Marie est bien celle qui commande "gloire" (vers elle montent les «psaumes» de l' «homme») et domine de "joie» toute «douleur».

Cet autre poème, du Suisse Eugen Gomringer montrera aussi quelle peut être la puissance expressive d'un silence, à partir d'un seul mot «Schweigen»:

\begin{tabular}{|c|c|}
\hline $\begin{array}{l}\text { schweigen } \\
\text { schweigen } \\
\text { schweigen }\end{array}$ & $\begin{array}{l}\text { schweigen } \\
\text { schweigen }\end{array}$ \\
\hline schweigen & schweigen \\
\hline
\end{tabular}

Ce poème est autre chose qu'un exercice de style ou même qu'une simple expérimentation. Déjà en réduisant la majuscule - obligatoire pour un substantif en allemand - l'auteur fait hésiter ce mot entre sa signification nominale ( $=$ silence) et verbale ( $=$ se taire). Mais surtout il introduit un silence spatial qui n'eût été que sémantique, une simple expression de silence, s'il avait opté pour la seule pause en décalage de vers et eût risqué d'aller à l'encontre de l'effet recherché, c'est-à-dire en suggérant une suite inachevée et indéfinie de silence:

schweigen schweigen
schweigen

En entourant quatorze fois de silence le blanc central, il donne à l'ensemble une densité que seule pouvait rendre une symétrie graphique et phonique, et où il apparaît que le chiffre 7 prend du même coup une résonance mystérieuse. Le silence est à la fois harmonique puisqu'il unit deux groupes symétriques. II est aussi isolant, fermé sur lui-même. La spatialisation a donc ajouté à la simple fonction vocative du mot une valeur concrète: le silence est désormais redevenu ce qu'il est essentiellement, non plus seulement une qualité expressive, mais une quantité d'espace visuel et sonore, à la limite un espace qui ne signifie rien d'autre que lui-même, un temps de silence qui ne renvoie qu'à lui-même.

La conclusion qui s'impose est grave. Dans l'univers poétique où il se meut, le poète moderne a retrouvé le sens du vertigineux, autant par les mots-matière par lesquels il désigne cet univers que par les silences contre lesquels il est contraint de -se heurter. On a jusqu'à présent fait la part trop belle à la dimension énergétique du poème, enfanteur de pos- 
sibles infinis. Le poète, de tout temps, s'est vu mage ou démiurge. Même Henri Chopin, dans sa réponse à Position I du Mouvement international (de poésie), manifeste qu'il n'a pas voulu signer, déclare: "L'artiste est vertigineux désormais. II est mouvement-combustion avec la combustion universelle. " Mais le silence auquel il se heurte en le créant, semble-t-il, de ses seules forces imaginatives, est là pour lui rappeler la nécessité de l'humilité. Car le silence fait partie de l'univers et pas seulement de l'homme. L'immense mérite de la poésie contemporaine est peut-être d'avoir rencontré une des composantes de tout acte créateur, qu'il soit de Dieu ou des hommes, le silence.

Pour en donner témoignage, relisons ce poème de Paul Chamberland, où nous retrouvons les trois "signes" essentiels du silence: sémantique, phonétique et géométrique.

\section{Poème d'appartenance}

retourné au nu langage

à ton virage ô terre égal à mon silence

à ma naissance à mon retour au profond de ton âge

à la vérité du labour de la biche sertie du

sommeil des forêts

et de la bête brune qui bêle renversée d'amour

sous le dieu immédiat

ô mère et ma propriété ma substance abîme

murmurant sous l'écume des mots

je te rends nu mon corps

crible sa nuit de sèves

(P. Chamberland, Terre Québec, Montréal, Déom, 1966)

Jean-Paul Mauranges

Université Laval

1. Sauf indication contraire, les textes sont extraits de Poésie du Québec, Anthologie, Montréal, $\mathrm{HMH}, 1971$, par Alain Bosquet. 